Juan Ignacio Leo-Castela* José Ignacio Sánchez Macías*"

Recibido: 5 de noviembre de 2019

Evaluación: 10 de noviembre de 2020

Aprobado: 12 de noviembre de 2020

Artículo de investigación

(C) 2021 Universidad Católica de Colombia. Facultad de Ciencias Económicas y Administrativas. Todos los derechos reservados

* Doctor Internacional en Economía Aplicada. Profesor asociado, doctor e investigador en el Departamento de Economía Aplicada. Correo electrónico: leocastela@usal.es. (D) https://orcid.org/0000-0003-2936-6017.

** Doctor en Economía y Abogado. Profesor Titular y Director del Master en Derecho y Políticas Públicas de la Universidad de Salamanca, Salamanca, España. Correo electrónico: macias@usal.es. (D) https://orcid.org/0000-0002-0081-1925.
Revista Finanzas y Política Económica, Vol. 13, N. ${ }^{\circ}$ 1, enero-junio, 2021, pp. 143-170 https://doi.org/10.14718/revfinanzpolitecon.v13.n1.2021.7

\title{
Las políticas de integridad corporativa como política económica en la OCDE ${ }^{1}$
}

\section{Resumen}

Durante el siglo XX, la doctrina económica tradicional se ha centrado en estudiar, desde distintas perspectivas, la relación entre la corrupción y los delitos económicos afines y el crecimiento y el desarrollo económico. Sin embargo, las nuevas políticas económicas y recomendaciones de la Organización para la Cooperación y el Desarrollo Económicos (OCDE) apuestan claramente por un mayor protagonismo de los factores institucionales en la prevención del daño social inherente a los delitos de corrupción. En la metodología de este trabajo se aplica, por un lado, el modelo econométrico de regresión probit y, por otro, el método estadístico de análisis de conglomerados por vinculación completa, para ilustrar la importancia creciente del cumplimiento corporativo en la OCDE, especialmente a partir de la regulación legal de la responsabilidad de las personas jurídicas y de la designación de un oficial de cumplimiento. Los resultados de investigación sugieren que ha existido una expansión geográfica de esta política de integridad desde los países de tradición anglosajona hacia el resto de los países miembro de la OCDE; asimismo, que los aspectos

1 Los autores agradecen la ayuda financiera del proyecto PID2019. 107743RB-I00 del Programa Estatal de Generación de Conocimiento y Fortalecimiento Científico y Tecnológico, del Sistema de I+D+i, orientado a los retos de la sociedad del Ministerio de Ciencia e Innovación (España). Este trabajo ha sido realizado en el contexto del grupo de investigación reconocido Economía y Políticas Públicas y el Centro de Investigación para la Gobernanza Global (CIGG) de la Universidad de Salamanca (España). 
institucionales e historicistas están recuperando importancia en el funcionamiento de la economía mundial como palanca de cambio para la integridad en las corporaciones.

Palabras clave: compliance, corrupción, integridad corporativa, OCDE, responsabilidad.

JEL Classification: F02, F53, K14, O57

\title{
Corporate Integrity Policies as Economic Policy in the OCDE
}

\begin{abstract}
In the 20th century, traditional economic doctrine was focused on studying from different perspectives, the relationship between corruption (and related economic crimes) and growth and economic development. However, new OECD policies and recommendations clearly support a greater role of institutional factors in the prevention of social harm inherent in corruption offenses. Using an econometric analysis methodology (probit regression model) and a complete-linkage clustering approach, this paper shows the growing importance of corporate compliance in the OECD, especially, since the legal regulation of the liability of legal persons and the appointment of a compliance officer. Our research results suggest that this integrity policy underwent a geographical expansion from the Anglo-Saxon countries to the rest of the OECD member countries; additionally, institutional and historical aspects are regaining importance in the functioning of the world economy as a lever of change for corporate integrity.

Keywords: compliance, corruption, corporate integrity, OECD, liability of legal persons.
\end{abstract}




\section{INTRODUCCIÓN}

La corrupción, los delitos económicos afines y la falta de ética e integridad inciden de manera determinante en el funcionamiento de las instituciones, las administraciones públicas y las organizaciones, generando efectos perniciosos para el conjunto de la economía global. Partiendo de esta premisa, no han sido pocos los economistas que se han centrado en investigar cómo las diferencias en los niveles de regulación y desarrollo económico entre distintos países pueden estar explicadas por fallos en el control interno de estos fenómenos y por la implementación de políticas públicas escasamente efectivas en términos de probidad (Shleifer y Vishny, 1993, pp. 600-602). Tampoco faltan las opiniones que, atendiendo a factores estrictamente cuantitativos, afirman la existencia de un efecto directo entre la criminalidad y el crecimiento económico en el contexto internacional (Mauro, 1995, pp. 682-684).

Sin embargo, elementos como el monitoreo o la adopción de controles internos para la lucha contra la corrupción son ejemplos de que la prosperidad y el crecimiento económico no solo dependen de factores estrictamente cuantitativos (Rupasingha, Goetz y Freshwater, 2002, pp. 140-141). En sintonía con esta postura, cabe subrayar que el bienestar y el desarrollo económico dependen también de diferentes factores institucionales y cualitativos. De entre todos ellos, en este trabajo se pretende poner el acento - por la importancia creciente que han ido adquiriendo en los últimos años- en las políticas de cumplimiento normativo (en adelante compliance, por la relevancia internacional del concepto) como palanca de cambio para la articulación de una nueva política económica mundial. Esta concepción parte del hecho de considerar que los efectos negativos derivados de la criminalidad corporativa sobrepasan las fronteras de lo nacional y representan un desafío global que requiere, de manera irremediable, de la colaboración institucional internacional.

La imparable digitalización de los intercambios y el crecimiento cada vez más acelerado de los flujos comerciales en la economía internacional representan importantes ventajas para el desarrollo económico, pero, al tiempo, comportan una serie de riesgos estrechamente vinculados al aumento de la criminalidad transnacional. Estos riesgos no siempre se supervisan ni se gestionan correctamente a nivel interno en las organizaciones. En este sentido, factores institucionales como la transparencia, la ética y la integridad están siendo determinantes en la configuración de una política económica internacional cada vez más orientada hacia la reducción significativa del riesgo de corrupción y los delitos económicos conexos. Desde esta perspectiva, cabe 
sostener que la adopción de políticas públicas y decisiones institucionales y corporativas en materia de control interno para la lucha contra la criminalidad económica transnacional también contribuye favorablemente al desarrollo y al crecimiento económico (Mo, 2001, pp. 68-70).

En este panorama, la gestión del riesgo legal se presenta como una herramienta extraordinariamente útil. En pocas palabras, al monitorear el cumplimiento de los requisitos legales previamente exigidos, es posible reducir la probabilidad de incurrir en un hecho ilícito (tanto en el sector público como en el privado). En este sentido, la gestión eficiente del riesgo legal tendería a minimizar los efectos negativos generalmente asociados a la delincuencia económica. Ciertamente, la disuasión de la delincuencia transnacional requiere de medidas nacionales eficaces para evitar, prevenir y combatir adecuadamente estos delitos. Además, es preciso contar con un segundo factor de igual importancia: la promoción a nivel internacional de una cultura organizativa que fomente el comportamiento ético de las empresas y el compromiso con el cumplimiento de la ley. Las recientes reformas de la Organización para la Cooperación y el Desarrollo Económicos (OCDE) en materia de responsabilidad de las personas jurídicas están promoviendo una nueva configuración de la integridad corporativa en la economía mundial, sobre todo mediante el fomento de medidas internas de cumplimiento en sus Estados miembro, entre las que sobresale la designación de un oficial de cumplimiento o supervisor interno en las organizaciones. A pesar de que la "cultura de cumplimiento" representa un concepto aparentemente impreciso, se trata de un activo intangible cada vez más relevante cuyas manifestaciones concretas se abordarán continuación.

El reconocimiento en el plano legal de la responsabilidad de las personas colectivas se presenta como un elemento catalizador que permite integrar el debate sobre la prevención de los delitos corporativos en el panorama económico internacional (Razzano y Nelson, 2008, p. 1259). Después de casi cuarenta años de anomia en esta materia, la OCDE optó por introducir expresamente la responsabilidad de las personas jurídicas en la Convención Anticohecho de 1997, sobre la lucha contra el soborno de funcionarios públicos extranjeros en las transacciones comerciales internacionales (firmada en 1997 y entrada en vigor desde 1999). El soborno extranjero y los delitos económicos relacionados son probablemente los tipos penales más extendidos en la actividad económica transnacional. Por esta razón, la acción internacional de la OCDE se ha visto complementada por otros acuerdos internacionales como el Segundo Protocolo del Convenio sobre la Protección de los Intereses 
Financieros de las Comunidades Europeas, de 1997, o la Convención de las Naciones Unidas contra la Corrupción, de 2004.

En este escenario, la prevención, el control, el seguimiento interno y el compromiso con la ley representan una nueva forma de gobierno en instituciones y empresas (Griffith, 2016, p. 2076) que contribuye a contrarrestar los efectos perniciosos de la corrupción. Durante la mayor parte del siglo XX, en las economías de la OCDE era la propia sociedad civil la que asumía los costos del crimen corporativo casi de un modo irremediable. Sin embargo, con la llegada del siglo XXI, son precisamente las organizaciones y empresas las que se ven abocadas a asumir el costo de prevenir este daño social, a menudo, bajo el temor de incurrir en un costo mayor derivado de la apreciación de su responsabilidad penal. De ahí que cobre especial relevancia la designación de un controlador interno u oficial de cumplimiento que lidere y dirija la función de compliance en la organización.

En el marco de este nuevo paradigma, las políticas de cumplimiento constituyen un nuevo desafío para la economía mundial, al menos por dos razones principales. En primer lugar, en una economía global los riesgos legales y económicos (aun siendo internos) a menudo pueden extenderse con bastante rapidez hacia otras economías, propagando sus efectos en la escena internacional. En este sentido, parece lógico abogar por mecanismos internacionales de prevención compartidos. En segundo lugar, está surgiendo una nueva función organizativa que conduce a cambios en la estructura y en el funcionamiento interno de cualquier organización (pública o privada) (Bird y Park, 2016, pp. 203-205): la función de cumplimiento corporativo. Se trata de una función extraordinariamente útil para la lucha contra el daño social asociado a los delitos económicos; por ello, requiere de un personal cualificado (cuya selección será esencial), así como de los recursos, la financiación y la atención necesarios por parte de la alta dirección, los gobiernos y las instituciones.

En línea con lo enunciado, el objetivo principal de este trabajo es investigar la evolución de las políticas de integridad corporativa en la OCDE a partir de las decisiones de cumplimiento institucional vinculadas con la prevención de la delincuencia económica; especialmente, por cuanto se refiere a la decisión de designar a un Chief Compliance Officer (en adelante, $\mathrm{CCO}$ ) una vez reconocida legalmente la responsabilidad de las personas colectivas.

El objetivo secundario es investigar la existencia de posibles liderazgos en la implementación de las políticas de integridad corporativa entre los Estados miembro de la OCDE, así como las posibles influencias que este liderazgo pudiera estar 
Juan Ignacio Leo-Castela • José Ignacio Sánchez Macías

ejerciendo sobre la evolución de las políticas de integridad en otros Estados, teniendo en cuenta tanto factores económicos como institucionales.

\section{UN MARCO LEGAL EMERGENTE EN LAS ECONOMÍAS DE LA OCDE}

Uno de los aspectos más destacados del panorama iberoamericano es el incentivo que se les confiere a las organizaciones y empresas para que eleven sus estándares éticos y de cumplimiento. Este elemento ha podido apreciarse con bastante claridad, por ejemplo, en las últimas reformas del Código Penal español de 2010, 2015 y 2019; o en las nuevas legislaciones latinas en materia de responsabilidad legal de las personas jurídicas. Interesa aquí discutir si este hecho está teniendo algún impacto cuantificable en las economías de la OCDE en términos de mejora de integridad, para posteriormente poder avanzar hacia la efectividad de los incentivos legales como instrumento válido en la lucha contra la criminalidad económica.

Desde una perspectiva microeconómica, la gestión ineficaz del riesgo (reputacional y económico) asociado a la corrupción puede afectar, entre otros aspectos, a los procesos de toma de decisiones de los accionistas y las partes interesadas 0 al valor de mercado de una compañía. Como es sabido, todo esto tiene importantes efectos sobre la integridad y la ética empresarial (Agrawal y Cooper, 2016, pp. 38-40). A propósito de esta cuestión existe un fuerte debate sobre quién debe evaluar y monitorear a nivel interno los comportamientos humanos tanto en el sector público como en el privado.

En la escena internacional, algunos autores han tratado de dar respuesta a esta pregunta - por ejemplo, en el Reino Unido-, planteando la necesidad de contar con un organismo supervisor (Keay, 2014, pp. 282-284) o aludiendo a la importancia de detectar y atajar las situaciones de "incumplimiento sistemático" (Macneil, 2006, p. 486). Como se ha podido demostrar, por ejemplo, en la economía alemana, el cumplimiento de los códigos de conducta, las normas y las recomendaciones de buenas prácticas tiene un impacto positivo en la valoración de las acciones (Goncharov, Werner y Zimmermann, 2006, pp. 435-440). En el caso sueco, se ha podido constatar la existencia de una relación positiva entre el cumplimiento y los códigos de gobierno corporativo (o las desviaciones de sus reglas) y otros elementos como la estructura de propiedad, la organización interna o la duración de los contratos de los ejecutivos (Tagesson y Collin, 2016, pp. 274-277). 
Por otra parte, desde un punto de vista macroeconómico, estos riesgos parecen justificar la conveniencia de alcanzar un consenso internacional en materia de prevención de delitos. Y esto básicamente con la finalidad de evitar retrocesos en la economía mundial. El ejemplo más claro en la mayor parte de los países europeos y latinoamericanos son las recientes regulaciones sobre responsabilidad penal, civil o administrativa de las personas jurídicas, con las que parece estar emergiendo un nuevo marco normativo que está sirviendo como punto de apoyo para la implementación de una nueva política económica internacional basada en la integridad de las corporaciones.

Precisamente, reconociendo el papel esencial que desempeña la responsabilidad de la persona jurídica en la prevención del crimen transnacional, el artículo 2 de la Convención Anticohecho de la OCDE de 1997 establece que "cada Parte adoptará las medidas que sean necesarias, de conformidad con sus principios jurídicos, para establecer la responsabilidad de las personas jurídicas...." Del mismo modo, en virtud del artículo 3, cada Parte está obligada a regular sanciones penales efectivas, proporcionadas y disuasorias. Después de 1997, muchos países de la OCDE (y otros países asociados) han ido aplicando expansivamente la responsabilidad de las personas jurídicas no solo al soborno extranjero, sino a una gama cada vez más amplia de delitos. Se dibuja así un panorama que no está exento de divergencias teóricas y prácticas entre las diferentes jurisdicciones. En este sentido, una de las principales dificultades a las que se enfrenta este nuevo marco normativo es su armonización en la escena internacional.

En definitiva, el reconocimiento normativo de la responsabilidad legal de las organizaciones y empresas determinará de manera definitiva la prevención de la delincuencia en la economía internacional del siglo XXI. Consciente de esta nueva realidad, la OCDE ha destacado la existencia del CCO como elemento clave en la organización interna de las personas colectivas de cara a una mejor compensación de los efectos negativos derivados de los delitos económicos. Como se verá enseguida, con base en este argumento de la OCDE es posible explicar la importancia de la designación del CCO como factor institucional en las economías seleccionadas.

Por otro lado, no sobra referir aquí que los mecanismos de control interno y de divulgación voluntaria (denuncia de irregularidades) desempeñan un papel fundamental, especialmente cuando una empresa opera en sectores altamente regulados, como el financiero. Tal característica a menudo se encuentra tanto en las economías europeas como en los países asiáticos (Al Sawalqa, 2014, p. 377). 
En relación con la importancia creciente de los mecanismos de denuncia, el pensamiento económico toma un nuevo impulso en la era del cumplimiento, fundamentalmente cuando concibe que una sólida política corporativa de compliance facilita la denuncia de ilegalidades contra la "cultura del silencio", y esto ayudará considerablemente a los administradores y gerentes a gobernar adecuadamente cualquier tipo de organización. No sobra recordar que aún no desempeñando la función de un CCO, estos sujetos tienen la misión clave de colaborar con él en la tarea de construir una sólida estrategia de integridad organizacional (Verhezen, 2010, pp. 195-197).

Por su parte, las auditorías externas y la labor que puedan realizar los supervisores neutrales contribuyen a fortalecer el compromiso de la organización con la conquista de altos niveles de cumplimiento en relación con los valores éticos y normativos. Un tercero ajeno a la organización podría determinar su adecuación para cumplir y la orientación de las políticas y protocolos corporativos hacia un logro efectivo de la aplicación de la ley (Walker, 2014, pp. 217-219).

La contribución clave de la presente investigación consiste en mostrar que elementos institucionales y organizativos como la designación del CCO pueden activar un "efecto dominó" en las "buenas prácticas", con importantes efectos positivos para la economía mundial. A partir de la regulación de la responsabilidad legal de las personas jurídicas, una nueva espiral moral está involucrando comportamientos de los agentes económicos (públicos y privados) en todo el mundo. Las buenas acciones refuerzan los incentivos para resistir las tentaciones y con frecuencia contribuyen a construir un capital moral global (Dal Bó y Terviö, 2013, pp. 621-623). Sin duda, este factor representa un apoyo fundamental en la nueva estructura económica de la OCDE.

\section{Desarrollo de hipótesis}

Como lo muestra la literatura, el cumplimiento normativo y las cuestiones legales constituyen la segunda prioridad más fuerte en materia de gestión de riesgos (Hubbard, 2009, pp. 32-33), por delante incluso de las pérdidas económicas o de cualquier otro tipo de riesgo, y solo después de la selección de los mejores métodos y técnicas de análisis de riesgos.

El cumplimiento normativo es esencial no solo para mejorar la gestión de riesgos, sino además para crear valor en la organización (Manab, Kassim y Hussin, 2010, pp. 247-249). Al designar a un CCO, la empresa puede monitorear el cumplimiento y reducir con ello el riesgo global al que se enfrenta (en muchos casos, materializado 
posteriormente en sanciones), especialmente si considera las nuevas reformas legales, principios y recomendaciones de la OCDE en materia de responsabilidad legal de las personas jurídicas. Lo anterior también resulta beneficioso para los grupos de interés y, en consecuencia, contribuye a crear valor dentro de las organizaciones (Berenbeim, 2004, p. 17; Hoyt y Liebenberg, 2011, p. 813) y en el conjunto global de la economía.

Debido a la interrelación existente entre la gestión de riesgos, el control interno y el cumplimiento normativo, algunos autores (Racz, Weippl y Seufert, 2010, pp. 109-111; Steinberg, 2011, pp. 41-43; Nissen y Marekfia, 2013, pp. 3-5) utilizan el acrónimo GRC (Governance, Risk and Compliance) para referirse en términos generales a la cultura organizativa de prevención, gestión y respuesta corporativa al riesgo como elemento base de la integridad colectiva. En el modelo económico actual del neoliberalismo, las decisiones institucionales y las múltiples conexiones entre la moral y la economía son decisivas para obtener ganancias o pérdidas (económicas, reputacionales o sociales) (Bloom, 2017, pp. 21-23; Wang, 2015, pp. 55-57). El diseño e implementación de políticas de integridad como la designación de un CCO contribuyen claramente a evitar daños sociales y, en consecuencia, constituyen un indicador fiable de esta nueva forma de entender la relación entre las organizaciones y su entorno. No en vano, ya desde el siglo pasado se viene advirtiendo la importancia de cumplir las obligaciones legales como parte esencial de la responsabilidad social corporativa de las organizaciones con su entorno (Carroll, 1979, pp. 497-500).

La consideración de las personas colectivas como sujeto legalmente responsable constituye una de las decisiones político-económicas más importantes que se recuerden en los últimos años. Tradicionalmente, las personas jurídicas se conciben como sujetos inimputables, por lo que no existía prácticamente ningún tipo de incentivo para fomentar y aplicar normas y procedimientos sobre el nombramiento o la designación de controladores internos. A pesar de ello, en muchas economías la regulación de esta responsabilidad genera nuevos riesgos para la reputación de las organizaciones, particularmente en aquellos supuestos donde la ausencia de un CCO puede favorecer que el comportamiento corporativo se desvíe de las buenas prácticas recomendadas por el regulador (Miller, 2017, pp. 9-11).

Los nuevos costes sociales y de reputación están relacionados con consecuencias económicas y jurídicas, como el cierre de establecimientos, la prohibición temporal de celebrar contratos con las administraciones públicas, la asunción de costas judiciales e incluso la extinción y muerte de la propia entidad. Sin embargo, 
todas estas consecuencias son evitables desde la implementación de un sistema de cumplimiento razonablemente diseñado. Sistema que, aún en aquellas jurisdicciones donde no se exige por imperativo legal, suele incluir la figura de un alto directivo o jefe de cumplimiento (CCO).

En el derecho comparado, la verificación de medidas de responsabilidad proactiva incluye a menudo el nombramiento del CCO como factor clave. En consecuencia, si surgen nuevos riesgos derivados de la regulación legal de la responsabilidad de las personas colectivas, entonces la primera hipótesis expresada en forma alternativa es la siguiente.

H1a. La regulación de la responsabilidad de las personas jurídicas en las economías de la OCDE tiene un efecto positivo en la designación de un oficial de cumplimiento (CCO), ceteris paribus.

Respecto a esta primera hipótesis, el propósito aquí es evaluar la relevancia de los factores institucionales e historicistas en la maduración de las políticas de integridad en la OCDE en relación con algunas de las variables macroeconómicas clásicas (como el PIB per cápita o el volumen de exportaciones). En este sentido, conviene expresar una segunda hipótesis en forma alternativa.

H1b. Los factores institucionales e historicistas tienen un efecto positivo en la designación de un oficial de cumplimiento (CCO), ceteris paribus.

Finalmente, para dar respuesta a nuestro objetivo secundario de investigar la existencia de posibles liderazgos en la implementación de las políticas de integridad corporativa entre los Estados miembro de la OCDE, resulta fundamental atender no solo a los factores institucionales e historicistas de cada jurisdicción, sino - y más importante aún - a la existencia de posibles concomitancias o influencias entre Estados. Por ello, se plantea la segunda hipótesis expresada en forma alternativa como sigue.

H2. La juridificación del compliance en las economías de la OCDE tiene un efecto de vinculación completa desigual entre los diferentes territorios, ceteris paribus.

\section{METODOLOGÍA DE INVESTIGACIÓN}

Hasta la fecha, poco se sabe acerca del impacto que representa la responsabilidad legal de las personas jurídicas en la economía mundial y en las decisiones sobre 
gestión de riesgos. Con la finalidad de poder aportar algo de luz a esta cuestión desde un enfoque jurídico-económico, en este trabajo se trata de determinar si la regulación de la responsabilidad corporativa induce a las economías de la OCDE a tomar decisiones institucionales sobre la prevención de riesgos legales, lo cual, probablemente, también contribuiría a mejorar su integridad.

No son pocos los académicos que asumen que los gobiernos, las instituciones y los reguladores generan estímulos de autorregulación en las corporaciones y empresas (Ruhnka y Boerstler, 1998, pp. 316-318; Fairman y Yapp, 2005, pp. 508-510; Sethi, 2016, pp. 7-9). Tales estímulos suelen estar tradicionalmente estructurados desde la premisa de cumplir con estándares éticos, recomendaciones o buenas prácticas (soft law o reglas no vinculantes). La regulación de la responsabilidad de las personas colectivas tiene una influencia positiva en el comportamiento humano y en la integridad corporativa, puesto que - parece claro- en todos los casos subyace la idea de establecer patrones de conducta o pautas para el buen comportamiento. Por esta razón, este trabajo hace hincapié en el hecho de que la respuesta de las organizaciones y empresas a estos incentivos incluya el nombramiento de un CCO.

Para el desarrollo de este trabajo empírico se han recopilado datos sobre 47 jurisdicciones (correspondientes a países de los cinco continentes) y sus decisiones institucionales de acuerdo con el informe OECD Corporate Governance Factbook 2017 en materia de gobierno corporativo y responsabilidad legal de las personas jurídicas. Los datos incluidos en este informe anual pueden utilizarse para examinar los efectos de la responsabilidad de las personas colectivas en las decisiones institucionales nacionales. La muestra incluye a los miembro de la OCDE y a los países que actualmente participan en procedimientos de cooperación reforzada y de adhesión.

\section{Modelo empírico}

El modelo que se plantea a continuación parte de la asociación entre la regulación de la responsabilidad corporativa como un factor institucional (ya sea en la rama penal o en cualquier otra no penal: civil o administrativa) y la designación de un CCO como variable indicativa de la integridad en las personas colectivas. Para medir la regulación nacional de la responsabilidad corporativa se utilizan dos variables dicotómicas codificadas como sigue. La variable CRIMINAL representa la regulación penal de la responsabilidad de las personas colectivas y adopta el valor 1 en aquellos países donde se ha reconocido expresamente esta responsabilidad en una norma penal ( 0 en caso contrario). La variable ONOCRIMINAL representa la regulación 
no penal (principalmente civil y/o administrativa) y adopta el valor 1 en aquellos países donde se ha reconocido expresamente esta responsabilidad en una norma no penal (0 en caso contrario). La designación del CCO se representa como otra variable dicotómica que adopta el valor 1 en aquellos países que, con independencia de su denominación, hubieran incorporado esta figura en su sistema de compliance ( 0 en caso contrario).

Como se explicará más adelante, tanto en la redacción de la $\mathrm{H} 1$ como en la construcción de esta variable se ha optado por seguir el criterio marcado por la OCDE: en sentido amplio, se considera la designación del CCO tanto si esta responde a un imperativo legal como si allá donde no estuviera expresamente regulada por ley, a juicio de la propia OCDE, dicha designación hubiera obedecido a otros factores extralegales, por ejemplo, la práctica empresarial de cada jurisdicción (OCDE, 2017).

Con la finalidad de profundizar y agudizar los resultados de la estimación, se han incluido varias variables de control que pueden dividirse en dicotómicas y no dicotómicas. En el grupo de las primeras se encuentra la variable CLAW, prevista para identificar las economías de tradición anglosajona en el conjunto de la OCDE. Esta variable adopta el valor 1 cuando se trata de un país de tradición anglosajona y el valor de 0 en caso contrario. Junto a ella, la variable STAGE distingue los países que han adoptado reformas legales en materia de responsabilidad de las personas colectivas antes del 2001 (valor 1) de aquellos que las han adoptado a partir del 1 de enero del 2002 (valor 0). Por otro lado, en este primer grupo se han incorporado también dos variables que pretenden recoger el componente geográfico del estudio. La variable EUROPE para identificar a las economías europeas de la OCDE (valor 1) de las del resto del mundo (valor 0); y la variable LAC para identificar a las economías latinoamericanas de la OCDE (valor 1 ) de las del resto del mundo (valor 0 ). Mención especial requiere la última variable de control dicotómica: LLIMIT, con la cual se indica la existencia de reglas que atenúan y/o eximen la responsabilidad legal de las personas jurídicas, teniendo en cuenta exclusivamente las previsiones legales de cada ordenamiento nacional. Así, para aquellos modelos nacionales de compliance que cuentan con una regulación legal expresa en materia de atenuación o exoneración de responsabilidad legal de las personas jurídicas — esto es, para los que prevén un límite de responsabilidad o liability limit-, la variable LLIMIT adopta el valor 1 ( 0 en caso contrario).

El segundo grupo de las variables del control está formado por dos variables no dicotómicas y puramente económicas: por un lado, la variable OPEN, o apertura de 
la economía, con la que se intenta captar la importancia del comercio internacional en cada jurisdicción a partir de su volumen de exportaciones; por otro, la variable INCOME, para representar el nivel de desarrollo económico, medido en términos de PIB per cápita de acuerdo con la base de datos del Banco Mundial. Por último, cabe señalar que el presente estudio es transversal y que todas las variables utilizadas se refieren al año 2017.

Dada la primera hipótesis (H1a) según la cual, después de controlar otras variables conocidas, la regulación de la responsabilidad de las personas colectivas en las economías de la OCDE tiene un efecto positivo en la designación de un CCO, la especificación propuesta puede expresarse formalmente en la ecuación [1]:

$$
\operatorname{Pr}(\mathrm{Y}=1 \mid \mathrm{X})=\Phi(\beta 0+\beta 1 \mathrm{X}) \quad[1]
$$

La ecuación anterior se modela como una regresión probit. A pesar de que los coeficientes del modelo probit no pueden ser interpretados de una manera directa, no es menos cierto que sus signos sirven como una indicación fiable de la dirección de los efectos sobre la variable dependiente, lo cual es consistente con el objetivo establecido en la hipótesis H1a. Dada la naturaleza de las variables y el pequeño tamaño de la muestra, se han utilizado métodos robustos de estimación de errores estándar. Tanto el modelo planteado como las variables seleccionadas permiten que, con la estimación probit, se pueda contrastar también la hipótesis $H 1 b$ según la cual, después de controlar otras variables, los factores institucionales e historicistas tienen un efecto positivo en la designación de un oficial de cumplimiento (CCO).

Finalmente, para la contrastación de la segunda hipótesis (H2) según la cual la juridificación del compliance en las economías de la OCDE tiene un efecto de vinculación completa desigual entre los diferentes territorios, se aplicará un análisis de conglomerados, tal y como se muestra en el apartado que sigue.

\section{ANÁLISIS DE CLÚSTERES}

Dada la naturaleza internacional de la muestra utilizada, es interesante explorar de antemano si las observaciones de los países podrían clasificarse en grupos similares, aplicando un análisis de conglomerados (Anderberg, 2014, pp. 160-162; Silverman, 2018, pp. 130-132) a partir de las variables descritas anteriormente. Se trata de una herramienta estadística de gran utilidad para explorar las diferencias y similitudes en 
muestras heterogéneas, especialmente cuando las observaciones pueden clasificarse de acuerdo con varios criterios. El objetivo principal es agrupar las observaciones buscando la máxima homogeneidad dentro de cada grupo logrando, al tiempo, la máxima heterogeneidad entre todos los grupos.

A partir de los diversos métodos de agrupación jerárquica por conglomerados, en este trabajo se utiliza la vinculación completa, también llamada agrupación del vecino más lejano. Este método maximiza la discriminación en las observaciones ya que mide la proximidad entre dos grupos, calculando tanto la distancia entre sus elementos más distantes como la similitud entre los menos similares (Everitt et al., 2010, pp. 76-79).

$$
\mathrm{dG1}, \mathrm{G} 2=\max (\mathrm{di}, \mathrm{j}) ; \mathrm{i} \in \mathrm{G} 1, \mathrm{j} \in \mathrm{G} 2 .
$$

Entre las ventajas que presenta el método de vinculación completa, la principal es que los resultados de la agrupación pueden representarse convenientemente con un dendrograma, que permite visualizar tanto la secuencia de integración de la agrupación como la distancia entre todas las fusiones (Gráfica 1).

De acuerdo con el modelo de regresión probit expuesto, en el análisis de conglomerados se utilizan las variables CRIMINAL, ONOCRIMINAL, CLAW, STAGE, LLIMIT, INCOME y OPEN. Si bien el número final de grupos puede oscilar en función de la técnica de conglomerado que se utilice, lo cierto es que el resultado de aplicar el método de agrupamiento de vinculación completa (a juicio de los autores, el más adecuado para las características de esta muestra) arroja un total de cinco grupos:

- Grupo 1: Argentina, Chile, República Checa, Estonia, Grecia, Hungría, Italia, España, Portugal, República de Corea, Letonia, Lituania, Polonia, Arabia Saudita, República Eslovaca y Eslovenia.

- Grupo 2: Brasil, China, Colombia, Federación de Rusia, México, India, Indonesia, Sudáfrica y Turquía.

- Grupo 3: Alemania, Australia, Austria, Canadá, Hong Kong, Nueva Zelanda, Singapur, Reino Unido, Estados Unidos, Bélgica, Dinamarca, Finlandia, Francia, Países Bajos, Suecia, Israel y Japón.

- Grupo 4: Islandia, Irlanda, Noruega y Suiza.

- Grupo 5: Luxemburgo. 
Tabla 1.

Análisis de conglomerados

\begin{tabular}{|c|c|c|c|}
\hline Clúster & Freq. & Percent & Cum. \\
\hline 1 & 16,00 & 34,04 & 34,04 \\
\hline 2 & 9,00 & 19,15 & 53,19 \\
\hline 3 & 17,00 & 36,17 & 89,36 \\
\hline 4 & 4,00 & 8,51 & 97,87 \\
\hline 5 & 1,00 & 2,13 & 100,00 \\
\hline Total & 47 & 100,00 & \\
\hline
\end{tabular}

Fuente: análisis de autores a partir de base de datos propia.

Nota: tamaño muestral $=47$. Método de agrupación de enlaces completos

Este ejercicio de agrupación corrobora la existencia de dos grupos predominantes (Grupo 1 y Grupo 3). El Grupo 1 incluye las economías de Europa oriental y meridional (República Checa, Estonia, Letonia, Lituania, Polonia, Eslovenia, Eslovaquia, Grecia, Italia, Portugal o España) y solo dos países latinoamericanos (Argentina y Chile). Todos estos países han desarrollado sistemas de responsabilidad penal y no penal en el siglo XXI. Comparten elementos esenciales en relación con las normas de cumplimiento corporativo, como lo demuestra, en particular, la comparación entre Chile y España. Sin embargo, las economías del sur de Europa (Italia, Grecia, Portugal y España) son miembros fundadores de la OCDE con una regulación de la responsabilidad de las personas jurídicas bastante posterior al momento de su incorporación. En contraste, para los países de América Latina y Europa del este, las regulaciones sobre este tipo de responsabilidad tienden a coincidir con el momento de su incorporación a la OCDE.

Por otro lado, el Grupo 3 está compuesto por países de la Common Law (Reino Unido, Nueva Zelanda, Canadá, Estados Unidos, Australia, Singapur o Hong Kong) y países de Europa central (Austria, Bélgica, Dinamarca, Alemania, Francia y Holanda). Todas estas economías conservan las legislaciones más antiguas de la OCDE en materia de responsabilidad de la persona jurídica. En particular, los países de Common Law tienen una tradición significativa y una amplia trayectoria jurídica en esta materia.

Al margen de estos dos grupos principales, el tercero (Grupo 2) incluye una mezcla de países asiáticos y africanos de derecho consuetudinario (India, Indonesia y Sudáfrica) y países latinoamericanos (Brasil, Colombia y México). Los países del norte de Europa (Irlanda, Islandia, Noruega y Suiza) forman el Grupo 4; finalmente, Luxemburgo constituye un último grupo independiente. 
Gráfica 1.

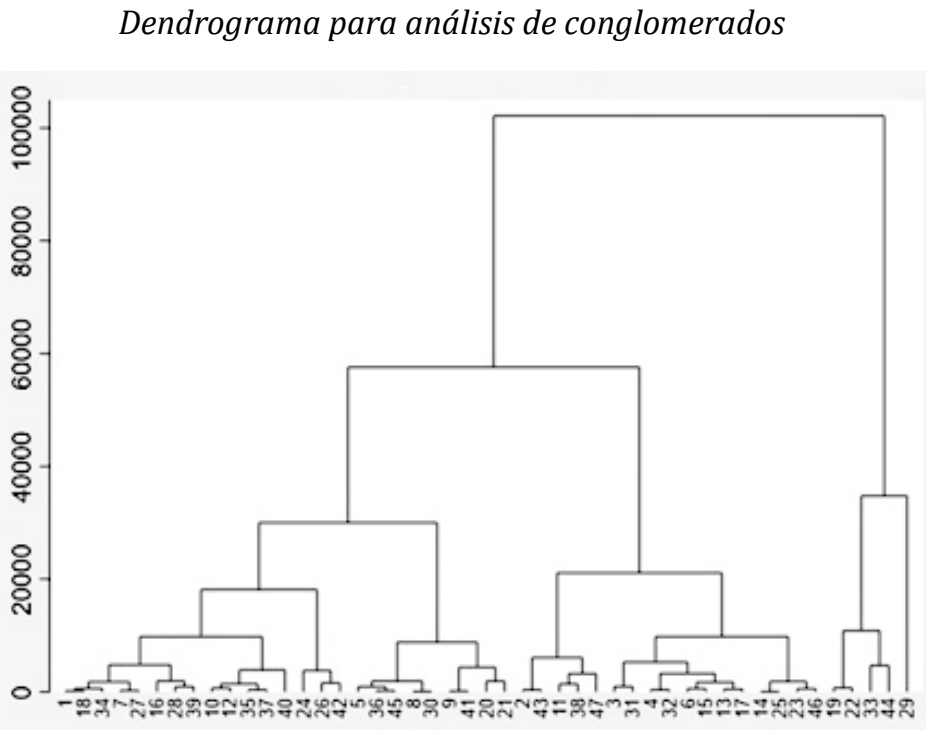

Fuente: elaboración de los autores.

Nota: el eje X representa las observaciones y la forma en que los cúmulos se están anidando. El eje Y representa el nivel de similitud (alternativamente, el nivel de distancia).

En vista de estos resultados, el análisis de conglomerados parece proporcionar una tendencia internacional fundamental del Common Law (con una gran tradición en el desarrollo de políticas de integridad relacionadas con la responsabilidad de la persona jurídica) sobre el resto de las economías de la OCDE. Con base en esto, los países europeos y latinoamericanos podrían haber recibido una influencia significativa de los países de Common Law a la hora de regular los principales requisitos legales en esta materia. No obstante, para profundizar en este argumento preliminar, en las siguientes secciones se relacionan estas conclusiones del análisis de conglomerados con los resultados econométricos del modelo estimado.

\section{Estadísticos descriptivos}

En este momento, la regulación de la responsabilidad de las personas colectivas presenta interesantes diferencias entre países. En tal sentido, el 85\% ha promulgado importantes reformas a las leyes corporativas utilizando la vía penal. Esta distribución porcentual es coherente con la tendencia al alza de la prevención de la delincuencia que se refleja en los tratados y consensos internacionales, como la Convención de 
Tabla 2.

Estadísticos descriptivos

\begin{tabular}{|c|c|c|c|c|c|}
\hline Variable & Mean & Median & $\begin{array}{c}\text { Standard } \\
\text { deviation }\end{array}$ & Min & Max \\
\hline CCO & 0,17 & 0,00 & 0,37 & 0,00 & 1,00 \\
\hline CRIMINAL & 0,85 & 1,00 & 0,35 & 0,00 & 1,00 \\
\hline ONOCRIMINAL & 0,19 & 0,00 & 0,39 & 0,00 & 1,00 \\
\hline CLAW & 0,23 & 0,00 & 0,42 & 0,00 & 1,00 \\
\hline STAGE & 0,51 & 1,00 & 0,50 & 0,00 & 1,00 \\
\hline EUROPE & 0,55 & 1,00 & 0,50 & 0,00 & 1,00 \\
\hline LAC & 0,10 & 0,00 & 0,311 & 0,00 & 1,00 \\
\hline OPEN & 53,76 & 37,90 & 46,39 & 7,94 & 230,00 \\
\hline LLIMIT & 0,42 & 0,00 & 0,49 & 0,00 & 1,00 \\
\hline INCOME & 29742,84 & 33805,00 & 23295,23 & 1939,61 & 104103,00 \\
\hline
\end{tabular}

Fuente: elaboración de los autores.

Nota: tamaño muestral $=47$ observaciones relativas a 47 Estados miembro de la OCDE y países asociados (ver Apéndice).

las Naciones Unidas contra la Corrupción de 2004 (capítulo III), la Directiva UE 2017/1371 (propuesta de 11 de julio de 2012) relativa a la lucha contra el fraude a los intereses financieros de la Unión mediante el derecho penal (artículo 6), o la Guía de la OCDE sobre buenas prácticas en materia de controles internos, ética y cumplimiento (adoptada el 18 de febrero de 2010).

Por supuesto, la alineación entre las regulaciones internacionales y nacionales en esta materia es una razón convincente para afirmar la existencia de mejoras significativas en la reducción de los delitos corporativos, sobre todo si se tienen en cuenta los riesgos inherentes al comercio internacional que se pretenden prevenir desde las normas de cumplimiento en el ámbito mundial. En esta línea, la prevención y la lucha contra la corrupción en las organizaciones es frecuentemente un objetivo compartido y de creciente importancia en la escena internacional (Kitchen y Schneider, 2004, pp. 92-95; Vlassis y Williams, 2013, p. 243; Crawford y Evans, 2017, p. 812).

\section{ANÁLISIS DE RESULTADOS}

La pregunta principal de esta investigación es si la regulación de la responsabilidad corporativa está asociada a la designación de un CCO como indicador de integridad 
Juan Ignacio Leo-Castela • José Ignacio Sánchez Macías

en las personas jurídicas. En la tabla 3 se muestran los resultados de la estimación de nuestro modelo probit.

Tabla 3.

Modelo de regresión probit, nombramiento de un oficial de cumplimiento

\begin{tabular}{|c|c|c|}
\hline Dep. Var. CCO. & Probit coef. & Marginal effects coef. \\
\hline CRIMINAL & $5,401^{* * *}$ & $0,785^{* * *}$ \\
& $(0,725)$ & $0,136)$ \\
\hline \multirow{2}{*}{ ONOCRIMINAL } & $5,898^{* * *}$ & $0,857^{* * *}$ \\
& $(0,655)$ & $0,131)$ \\
\hline CLAW & $1,876^{* *}$ & $(0,104)$ \\
\hline \multirow{2}{*}{ STAGE } & $(0,753)$ & $1,811^{* * *}$ \\
\hline \multirow{2}{*}{ EUROPE } & $12,454^{* * *}$ & $(0,278)$ \\
\hline \multirow{2}{*}{ LAC } & $(0,828)$ & 0,067 \\
& 0,461 & $(0,102)$ \\
\hline \multirow{2}{*}{ OPEN } & $(0,718)$ & $1,818^{* * *}$ \\
& $12,505^{* * *}$ & $(0,327)$ \\
\hline \multirow{2}{*}{ LLIMIT } & $(1,364)$ & 0,001 \\
& 0,008 & $(0,001)$ \\
\hline \multirow{2}{*}{ INCOME } & $(0,008)$ & 0,079 \\
& 0,549 & $(0,146)$ \\
\hline Observations & $(1,023)$ & $-8,18 \mathrm{e}-06^{* *}$ \\
\hline
\end{tabular}

Fuente: elaboración de los autores.

Notas: errores robustos entre paréntesis. Nivel de significación: ${ }^{* * *} \mathrm{p}<0,01,{ }^{* *} \mathrm{p}<0,05,{ }^{*} \mathrm{p}<0,1$. Wald chi-cuadrado $(9)=1468,38$. Pseudo R-cuadrado $=0,4237$.

La tabla 3 muestra un efecto positivo y significativo entre la regulación de la responsabilidad de las personas jurídicas (penal o no penal) y la decisión de nombrar a un CCO. Esta conclusión sugiere que el nombramiento del CCO puede entenderse como una respuesta institucional a una gestión adecuada del riesgo e igualmente como un resultado de la creciente importancia internacional que han adquirido los oficiales de cumplimiento normativo en los últimos años (Power, 2005). Conviene matizar en este punto que, conforme al nuevo criterio acogido por la OCDE, en la construcción de la variable dicotómica CCO se ha optado por considerar que esta adopte el valor 1 cuando el sistema nacional de compliance hubiera incorporado la designación del CCO tanto si dicha incorporación obedece a un imperativo legal 
como a la práctica empresarial habitual de cada jurisdicción bajo el criterio de la OCDE (2017).

Por otro lado, se encuentra un efecto positivo y significativo entre las economías de Common Law (CLAW), la existencia de legislaciones anteriores al cambio de siglo (STAGE), los países latinoamericanos (LAC) y el nombramiento de un CCO. Este resultado inicial es relevante sobre todo si se tiene en cuenta la escasez de investigaciones realizadas a propósito de la asociación entre la regulación de la responsabilidad de las empresas y la designación de oficiales de cumplimiento normativo en los países de la OCDE. En esta línea, es de gran interés examinar las diferentes legislaciones nacionales para identificar patrones comunes que puedan explicar estas tendencias internacionales. En este sentido, al profundizar en los sistemas legales de los países de la OCDE, se encuentra una tradición legal bastante arraigada, específicamente en los países de derecho consuetudinario, basada en el nombramiento de un responsable visible de la gestión del riesgo organizacional. Esta opinión se confirma a través de los resultados de la estimación de las variables CLAW y STAGE.

Es posible considerar que esta tradición jurídica tan característica del derecho anglosajón tiene efectos igualitarios en las nuevas normas de cumplimiento corporativo, en especial, en los recientes sistemas de responsabilidad corporativa latinoamericanos, promulgados principalmente en los últimos quince años. En este sentido, la Tabla 3 presenta los resultados de la estimación de la variable LAC de acuerdo con esta premisa.

Por otra parte, en la legislación nacional de los países europeos no es frecuente encontrar una referencia explícita al nombramiento de un oficial de cumplimiento en las normas de responsabilidad empresarial (penal o de cualquier otro tipo no penal). No obstante, ello no resulta un obstáculo para la discusión de resultados si se tiene en cuenta que la definición de la variable CCO no se limita exclusivamente a la imposición legal de contar con esta figura, como se indica en el apartado cuarto. A pesar de ello, las directrices europeas en materia de buenas prácticas y las recomendaciones institucionales y de soft law suelen contener menciones al papel e importancia que representa el CCO en el viejo continente, en consistencia con el concepto manejado por la OCDE. Ello puede estimular su inclusión en los códigos de conducta de organizaciones y empresas.

Asimismo, es imperante considerar el objetivo de asegurar la aplicación efectiva de los principios de buenas prácticas en los mercados bursátiles, con el fin de promover estándares de cumplimiento más exigentes para las sociedades 
cotizadas, ya que estas empresas suelen afrontar mayores niveles de riesgo como consecuencia de su mayor tamaño (volumen de activos o número de empleados), mayor complejidad (mayor diversificación) o mayor grado de internacionalización. Esta opinión es coherente con el requisito de establecer disposiciones específicas que describan las responsabilidades del órgano de gobierno en materia de gestión de riesgos. Así, en la revisión de las distintas legislaciones de los Estados miembro de la OCDE se halla que la designación de un CCO no es requerida por las normas nacionales de cotización. Curiosamente, esta posición parece ser una característica común en aquellas empresas que cotizan en bolsa, lo cual puede servir como base para la realización de futuras investigaciones en esta materia.

No debe sorprender que, además de la responsabilidad penal de las personas jurídicas, el nuevo marco normativo establezca con carácter general el catálogo de responsabilidades y obligaciones en materia de gestión de riesgos. En tal sentido, los sistemas de cumplimiento se adaptan principalmente a los requisitos legales o a las buenas prácticas que avalan los reguladores o las resoluciones judiciales. En muchos casos se concibe que las circunstancias que pueden eximir o atenuar la responsabilidad de una empresa dependen casi exclusivamente del nivel de cumplimiento de estas normas. Así, la promulgación de una nueva responsabilidad legal corporativa refuerza la percepción del cumplimiento dentro de las organizaciones y fomenta la idea de que el sistema de cumplimiento puede tener una importancia considerable. En otras palabras, establecer estas responsabilidades como meras recomendaciones puede ser menos efectivo en el objetivo de modificar comportamientos corporativos. En consecuencia, puede argumentarse que la regulación de nuevas responsabilidades para la gestión de riesgos sirve como estímulo adicional para elevar el grado de cumplimiento, probablemente a niveles mucho más altos de lo esperado. Tales incentivos no solo fomentan la autorregulación empresarial, sino también la colaboración privada en la lucha contra desórdenes públicos y en la prevención de la delincuencia, facilitando la investigación de hechos ilícitos "allá donde el Estado no llega".

Los resultados de la Tabla 3 sugieren que la regulación es la opción preferida para incluir el nombramiento de un CCO dentro de las responsabilidades de gestión de riesgos, ampliando las funciones previstas en los códigos de conducta internos, protocolos u otras normas no vinculantes que, sin embargo, podrían complementar a las normas jurídicas. Este argumento es consistente con la idea de que la gestión de riesgos es una función de extraordinaria importancia tanto para las corporaciones como para la sociedad en su conjunto, pues, al fin y al cabo, la sociedad es la 
principal perjudicada por los delitos corporativos. No se puede dejar de referir en este punto que, aunque se trate de una premisa cualitativa que aún no se ha podido contrastar empíricamente, el nombramiento de supervisores de cumplimiento y legalidad comienza a perfilarse en el panorama internacional como un ingrediente fundamental en la configuración de la integridad corporativa. Tal aspecto, sin duda, puede resultar interesante de explorar en futuras investigaciones. En consonancia, las empresas han reconocido los efectos positivos de las normas de responsabilidad penal, sobre todo al tener en cuenta la anomia anterior en la mayor parte de los países de la OCDE.

Puede entonces argumentarse con suficiencia que este hallazgo es estadísticamente significativo y sustancial desde un punto de vista jurídico y económico, pues existe un apoyo razonable para la hipótesis H1 (la regulación de la responsabilidad de las personas jurídicas en las economías de la OCDE tiene un efecto positivo en el nombramiento de un oficial de cumplimiento).

La tabla 3 muestra los resultados de la regresión probit, donde los coeficientes de las variables CRIMINAL, ONOCRIMINAL, CLAW, STAGE, LAC y LLIMIT son altamente significativos y ejercen una influencia positiva en la designación de un CCO. Asimismo, el modelo tiene un respetable poder predictivo, con un pseudo R-cuadrado del $42 \%$, el cual presenta como un resultado el test de Wald igualmente satisfactorio. Todo ello indicaría que la validez del modelo es satisfactoria para el conjunto de las variables estudiadas.

\section{CONCLUSIONES}

Las instituciones, empresas, organizaciones y personas colectivas en general desempeñan un papel indiscutible en la economía mundial como actores del mercado y agentes sociales. Este hecho permite considerar que su estructura y características también las convierte en agentes idóneos para la prevención del daño social procedente de determinados delitos (en particular, delitos económicos y delitos conexos).

Desde esta perspectiva, la idea de involucrar a las personas jurídicas en la prevención del delito a nivel internacional supone que estas tienen la capacidad interna para detectar y prevenir actos ilícitos mediante la adopción y el desarrollo de nuevas estructuras y formas de gobernanza. Sin embargo, en términos generales, no es probable que una organización tenga suficientes incentivos para adoptar controles internos y medidas de cumplimiento que refuercen su integridad a menos 
que perciba un riesgo de incurrir en ciertos tipos de pérdidas (económicas, sociales, reputacionales...). Precisamente, el artículo 2 de la Convención de la OCDE contra el soborno (1997) introduce este incentivo cuando establece la responsabilidad de las personas colectivas como un instrumento para la prevención del delito. En otras palabras, establece que la regulación de esta responsabilidad apoya, refuerza y fortalece la lucha contra los retos económicos mundiales, como la actividad delictiva transfronteriza y la prevención de la delincuencia.

En primer lugar, el impulso de la OCDE a las reformas legales que conciben la responsabilidad de la persona colectiva como un instrumento adecuado para prevenir los delitos de soborno internacional ha propiciado cambios en la prevención de la delincuencia pública y privada, en particular a través del nombramiento de un CCO. Tal situación, por otro lado, representa un importante avance en el camino hacia la consolidación de las políticas de integridad corporativa en el contexto de la economía global.

Los resultados de este trabajo sugieren que hay razones suficientes para argumentar que la tradición jurídica más arraigada en los países de Common Law está desempeñando un papel clave en las economías de la OCDE, en específico como fuente de inspiración para las normas de cumplimiento más recientes aprobadas en Europa y América Latina, contribuyendo así a la expansión de la responsabilidad de las personas jurídicas a nivel internacional y obstaculizando la impunidad de los delitos corporativos transnacionales.

Las ventajas derivadas de esta expansión de la cultura anglosajona de cumplimiento no solo se circunscriben al ámbito penal, administrativo o civil, sino que cada vez más empiezan a observarse en otros campos del derecho y de la economía. Tal es el caso, por ejemplo, del cumplimiento tributario (Evans y Walpole, 1999, p. 111; Andreoni, Erard y Feinstein, 1998, pp. 849-854), donde se han abordado ideas de gran interés como los acuerdos de liquidación, la elaboración de perfiles fiscales o la mejora de las relaciones entre el particular y la Administración (todas ellas, distintas manifestaciones del llamado cumplimiento cooperativo). Algunos de estos conceptos se recogían, años atrás, en el Estudio de la OCDE de 2009 sobre prácticas de control y aplicación de la gobernanza empresarial en los Estados miembro. Además, la clasificación de los contribuyentes en función de su grado de cumplimiento está incluida en el nuevo proyecto de Directiva Europea de 2022 en materia del impuesto sobre el valor añadido.

La experiencia acumulada de los países de Common Law en materia de cumplimiento se está afianzando en el contexto internacional, y no exclusivamente en los ámbitos tradicionales (como la fiscalidad internacional), sino también en otros más 
recientes, como los retos empresariales y la responsabilidad de las personas colectivas en sentido amplio. En esta línea, desde un enfoque jurídico-económico es posible afirmar que las políticas de cumplimiento como una nueva forma de construir integridad y de proteger el buen funcionamiento de la economía global han llegado para quedarse.

En segundo lugar, mientras que las variables institucionales del modelo probit son bastante significativas, las económicas no lo son (variable INCOME y variable OPEN). Esto, una vez más, pone de relieve la importancia de los aspectos institucionales e historicistas en el funcionamiento de la economía mundial. Como indica la OCDE, la regulación de la designación de un CCO (independiente, fiable, con formación específica, etc.) es un fuerte indicador del compromiso ético y de la integridad de los Estados miembro.

Como se muestra en este trabajo, en este primer enfoque las economías más ricas o las que concentran los mayores flujos comerciales internacionales no son precisamente

las más propensas a reforzar un compromiso ético de probidad y de respeto a la ley. Por el contrario, aquellas que, con independencia de su nivel de riqueza o de participación en la actividad económica internacional, han desarrollado aspectos institucionales vinculados con la integridad corporativa, sí reflejan este compromiso, particularmente cuando entre esos elementos institucionales se incluye la adopción de reformas legales relativas a la responsabilidad de la persona jurídica (penal o no penal). En esta aspecto, la influencia anglosajona en las economías de la OCDE está teniendo una relevancia cada vez mayor.

\section{REFERENCIAS}

1. Agrawal, A. y Cooper, T. (2016). Corporate governance consequences of accounting scandals: evidence from top management, CFO and auditor turnover. Quarterly Journal of Finance, 7, 1-41.

2. Al Sawalqa, F. (2014). Corporate governance mechanisms and voluntary disclosure compliance: the case of banks in Jordan. International Journal of Academic Research in Accounting, Finance and Management Sciences, 4, 369-384.

3. Anderberg, M. R. (2014). Cluster analysis for applications: probability and mathematical statistics: a series of monographs and textbooks. Londres: Academic Press.

4. Andreoni, J., Erard, B. y Feinstein, J. (1998). Tax compliance. Journal of Economic Literature, 36(2), 818-860. 
5. Berenbeim, R. (2004, 27-29 de octubre). The value based enterprise: A new corporate citizenship paradigm. Paper presented at The Asia Foundation's Hong Kong Symposium on Corporate Citizenship and the Taipei Corporate Citizenship Forum, Hong Kong.

6. Bird, R. C. y Park, S. K. (2016). The domains of corporate counsel in an era of compliance". American Business Law Journal, 53, 203-249.

7. Bloom, P. (2017). The ethics of neoliberalism: the business of making capitalism moral. Nueva York: Routledge.

8. Carroll, A. B. (1979). A three-dimensional conceptual model of corporate performance. Academy of Management Review, 4(4), 497-505.

9. Crawford, A. y Evans, K. (2017). Crime prevention and community safety. Oxford: Oxford University Press.

10. Dal Bó, E. y Terviö, M. (2013). Self-esteem, moral capital, and wrongdoing. Journal of the European Economic Association, 11(3), 599-633.

11. Evans, C. y Walpole, M. (1999). Compliance cost control: A review of Tax Impact Statements in the OECD. Australian Tax Research Foundation Research Studies, 115.

12. Everit, B., Landau, S., Leese, M. y Stahl, D. (2010). Cluster analysis. Londres: John Wiley \& Sons.

13. Everitt, B., Landau, S., Leese, M. y Stahl, D. (2001). Cluster analysis (4. $\left.{ }^{\mathrm{a}} \mathrm{ed}\right)$. Londres: Arnold.

14. Fairman, R. y Yapp, C. (2005). Enforced self-regulation, prescription, and conceptions of compliance within small businesses: The impact of enforcement. Law $\mathcal{E}$ Policy, 27(4), 491-519.

15. Goncharov, I. Werner, J. R. y Zimmermann, J. (2006). Does compliance with the German corporate governance code have an impact on stock valuation? An empirical analysis. Corporate Governance: An International Review, 14, 432-445.

16. Griffith, S. J. (2016). Corporate governance in an era of compliance. William and Mary Law Review, 57, 2075-2140.

17. Hoyt, R. E. y Liebenberg, A. P. (2011). The value of enterprise risk management. Journal of Risk and Insurance, 78(4), 795-822.

18. Hubbard, D. W. (2009). The failure of risk management: Why it's broken and how to fix it. Nueva Jersey: John Wiley \& Sons.

19. Keay, A. (2014). Comply or explain in corporate governance codes: in need of greater regulatory oversight? Legal Studies, 34, 279-304.

20. Kitchen, T. y Schneider, R. H. (2004). Planning for crime prevention: A transatlantic perspective. Londres: Routledge.

21. Macneil, I. (2006). Comply or explain: Market discipline and non-compliance with the Combined Code. Corporate Governance: An International Review, 14, 486-496. 
22. Manab, N. A., Kassim, I. y Hussin, M. R. (2010). Enterprise-wide risk management (EWRM) practices: Between corporate governance compliance and value. International Review of Business Research Papers, 6(2), 239-252.

23. Mauro, P. (1995). Corruption and growth. The Quarterly Journal of Economics, 110(3), 681-712.

24. Miller, G. P. (2017). The law of governance, risk management, and compliance. Nueva York: Wolters Kluwer Law \& Business.

25. Mo, P. H. (2001). Corruption and economic growth. Journal of Comparative Economics, 29(1), 66-79.

26. Nissen, V. y Marekfia, W. (2013). Towards a research agenda for strategic governance, risk and compliance (GRC) management. En Business Informatics (CBI), 2013 IEEE $15^{\text {th }}$ Conference on (pp. 1-6).

27. Organización para la Cooperación y el Desarrollo Económicos (OCDE). (2007) Convención Anticohecho de la OCDE 1997. https:/www.oecd.org/daf/anti-bribery/ ConvCombatBribery_Spanish.pdf

28. Organización para la Cooperación y el Desarrollo Económicos (OCDE) (2017). OECD Corporate Governance Factbook 2017. https://www.oecd.org/daf/ca/OECD-CorporateGovernance-Factbook-2017.pdf

29. Power, M. (2004). The risk management of everything. The Journal of Risk Finance, $5(3), 58-65$.

30. Power, M. (2005). Organizational responses to risk: The rise of the chief risk officer. En H. Bridget y M. Power (Eds.), Organizational encounters with risk (pp. 132-148.). Cambridge: Cambridge University Press.

31. Racz, N., Weippl, E. y Seufert, A. (2010). A frame of reference for research of integrated governance, risk and compliance (GRC). En IFIP International Conference on Communications and Multimedia Security (pp. 106-117). Berlín: Springer.

32. Razzano, F. C. y Nelson, T. P. (2008). The expanding criminalization of transnational bribery: Global prosecution necessitates global compliance. Int'l Law., 42, 1259.

33. Ruhnka, J. C. y Boerstler, H. (1998). Governmental incentives for corporate selfregulation. Journal of Business Ethics, 17(3), 309-326.

34. Rupasingha, A., Goetz, S. J. y Freshwater, D. (2002). Social and institutional factors as determinants of economic growth: Evidence from the United States counties. Papers in Regional Science, 81 (2), 139-155.

35. Sethi, S. (2016). Globalization and self-regulation: The crucial role that corporate codes of conduct play in global business. Nueva York: Springer.

36. Shleifer, A. y Vishny, R. W. (1993). Corruption. The Quarterly Journal of Economics, 108(3), 599-617.

37. Silverman, B. W. (2018). Density estimation for statistics and data analysis. Londres: Routledge. 
38. Steinberg, R. M. (2011). Governance, risk management, and compliance: It can't happen to us-avoiding corporate disaster while driving success. Nueva York: John Wiley \& Sons.

39. Tagesson, T. y Collin, S. Y. (2016). Corporate governance influencing compliance with the Swedish code of corporate governance. International Journal of Disclosure and Governance, 13, 262-277.

40. Verhezen, P. (2010). Giving voice in a culture of silence. From a culture of compliance to a culture of integrity. Journal of Business Ethics, 96, 187-206.

41. Vlassis, D. y Williams, P. (2013). Combating transnational crime: Concepts, activities and responses. Londres: Routledge.

42. Walker, C. (2014). Organizational learning: The role of third party auditors in building compliance and enforcement capability. International Journal of Auditing, 18, 213-222.

43. Wang, X. (2015). On moral capital. Londres: Springer.

\section{APÉNDICE}

1. Regulación de la responsabilidad de la persona jurídica en las economías de la OCDE

2. Argentina. Ley 27401, de 8 de noviembre de 2017, que modifica el Código Penal y el Código Procesal Penal de la Nación, y establece el régimen de responsabilidad penal aplicable a las personas jurídicas privadas por determinados delitos.

3. Australia. Criminal Code Act 1995 (consolidated as of July 1, 2017).

4. Austria. 2006 Bundesgesetz über Verantwortlichkeit von Verbänden für Straftaten - Verbandsverantwortlichkeitsgesetz (VbVG). Austrian Federal Statute on the Responsibility of Entities for Criminal Offences (2006).

5. Belgium. 8 juin 1867 - Code pénal, réforme de 1999 (mise à jour le 1er janvier 2012).

6. Brazil. Lei 12.846, de 1을 de agosto de 2013. Dispõe sobre a responsabilização administrativa e civil de pessoas jurídicas pela prática de atos contra a administração pública, nacional ou estrangeira, e dá outras providências. Entrada em vigor em 2014.

7. Canada. Criminal Code (R.S.C., 1985, c. C-46). Corruption of Foreign Public.

8. Chile. Ley 20.393 de 2009 sobre responsabilidad penal de la persona jurídica por los delitos que tipifica.

9. China. Chinese criminal code (1977).

10. Colombia. Ley 1437 de 2011. Ley 1778 de 2016, sobre responsabilidad administrativa de la persona jurídica.

11. Czech Republic. Law 418 / 2011 Chapter 146. 418 Act of 27 october 2011 on criminal liability of legal persons and proceedings against them (418/2011 Sb., o trestní odpovědnosti právnických osob a řízení proti nim nim). Entry into force in 2012.

12. Denmark. Butler's Law of 1926; Amendments of the Penal Code of 1996 and 2002. 
13. Estonia. Penal code (reform 2002). (Karistusseadustik Vastu võetud 06.06.2001 RT I 2001, 61, 364 jõustumine 01.09.2002).

14. Finland. Criminal law 1995 and criminal code (2003 reform), Chapter 9, section 4.

15. France. Code pénal (Amendement de 1994). Sapin II Law (2016).

16. Germany. Administrative liability for corruption since 1994 (Munich I Public Prosecutor's Office, Anti-Corruption Division (Division XII). Act on Regulatory Offenses (Ordnungswidrigkeitengesetz or OWiG), section 30. Criminal Code (Strafgesetzbuch or StGB).

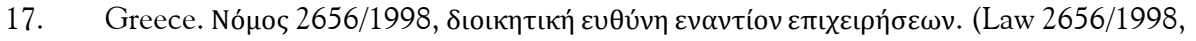
administrative liability against enterprises (not all legal persons), article 5). Civil code ( $\alpha \sigma \tau$

18. Hong Kong. Prevention of Bribery Ordinance, 1999. Cap 201 ("POBO”). Companies Ordinance and Criminal Procedure Ordinance.

19. Hungary. Law 104/2001 of criminal measures against legal entities.

20. Iceland. General Penal Code, Nr. 19/1940 (reform 1998). Also, previous forms of LP liability in specialised laws (customs and tax).

21. India. Indian Companies Act of 1956. Section 168.

22. Indonesia. Law 31 of 1999 on the Eradication of Corruption as lastly amended by Law 20 of 2001 ("Anti-Corruption Law"). Law 32/2009 on Environmental Protection and Management.

23. Ireland. 1937 Interpretation Act and subsequent laws. Criminal Justice Act (2018). Companies Acts 1963-2013, Limited Partnerships Act 1907, Investment funds, companies and miscellaneous provisions Act 2006.

24. Israel. 1994 Criminal Code reform. Companies Law 1999.

25. Italy. Decreto Legislativo 8 giugno 2001, n. 231 "Disciplina della responsabilita' amministrativa delle persone giuridiche, delle societa' e delle associazioni anche prive di personalita' giuridica, a norma dell'articolo 11 della legge 29 settembre 2000, n. 300". Pubblicato nella Gazzetta Ufficiale n. 140 del 19 giugno 2001.

26. Japan. Labour Standards Act (労働基準法, 1947). Japan's Whistleblower Protection Act (2006). The Act on the Protection of Personal Information (Act 57 of 2003, Enforced on 1 January 2016, Amendment: Act 65 of 2015), Unfair Competition Prevention Act (Act 47 of May 19, 1993, as amended up to Act 54 of July 10, 2015).

27. Latvia. Amendments to the Criminal Law (5 of May of 2005 and 14 March 2013).

28. Lithuania. Criminal Code reform 2002. (Last amended on 23 April 2015). Law on Companies of the Republic of Lithuania, 13 July 2000 VIII-1835. Companies Act 2001.

29. Luxembourg. Loi du 3 mars 2010, "Responsabilité pénale des personnes morales". Code Pénal (2016).

30. Mexico. Código Nacional de Procedimientos Penales, 2014. Código Penal Federal (última reforma, 21 de junio de 2018). 
Lorena Sotelo Forero • José Ignacio Sánchez Macías

31. Netherlands. Criminal Code (1976), paragraph 51. Code of Criminal Procedure, 2012.

32. New Zealand. Crimes Act 1961. Interpretation Act 1999. New Zealand Bill of Rights Act 1990.

33. Norway. General Penal Code (1997 reform).

34. Officials Act S.C. 1998, (CFPOA) c. 34.

35. Poland. Act of 28 october 2002 on liability of collective entities for acts prohibited under penalty (Ustawa z dnia 28 października 2002 r. O odpowiedzialności podmiotów zbiorowych za czynności zabronione pod groźbą kary).

36. Portugal. Reforma do Código Penal (2007).

37. Republic of Korea. Act on Preventing Bribery of Foreign Public Officials in International Business Transactions (FBPA, 1999). Anti-corruption Act No. 8878, Feb. 29, 2008.

38. Russian Federation. Federal Law No. 196-FZ of December 30, 2001 of the Enactment of the Code of Administrative Offences of the Russian Federation (Кодекс Российской Федерации об административных правонарушениях, frequently abbreviated КоАП РФ). Article 14 of Federal Law 273-FZ of 25 December 2008 On Counteracting Corruption and Article 19.28 of the Code of Administrative Offences.

39. Saudi Arabia. Law for Combatting Bribery issued pursuant to Royal Decree M/36 dated 29/12/1412H (corresponding to 01/06/1992G). The Anti-Money Laundering Law, issued pursuant to Royal Decree M/39 dated 25/06/1424H (corresponding to 23/08/2003G). The Public Environmental Law, issued pursuant to Royal Decree M/34 dated 28/07/1422H (corresponding to $15 / 10 / 2001 \mathrm{G})$.

40. Singapore. Penal Code. Original Enactment: Ordinance 4 of 1871. Revised edition 30th November 2008. Companies Act. Original Enactment: Act 42 of 1967. Revised edition 2006.

41. Slovak Republic. Act 91/2016 Coll. on Criminal Liability of Legal Persons (Zakon št. 91/2016 Coll. o kazenski odgovornosti pravnih oseb).

42. Slovenia. Penal Code (1999 reform). (Kazenski zakonik, spremenjen leta 1999).

43. South Africa. Criminal Procedure Law 51 of 1977, section 332.

44. Spain. Ley Orgánica 1/2015, de 30 de marzo, por la que se modifica la Ley Orgánica 10/1995, de 23 de noviembre, del Código Penal.

45. Sweden. Companies Act 2005, section 42. (New provision on negligent financing of bribery entered into force in 2012).

46. Switzerland. 2003 Criminal Code reform.

47. Turkey. Criminal Code Law Nr. 5237, last reform (26 of september of 2004).

48. United Kingdom. 1889 Public Bodies Corrupt Practices Act. 1906 Prevention of the Corruption Act. Criminal Law Act 1967. Bribery Act 2010.

49. United States of America. The Foreign Corrupt Practices Act of 1977 (FCPA) (15 U.S.C. § 78dd-1). 